\title{
THE PEAK STRENGTH OF SAND-STEEL INTERFACES AND THE ROLE OF DILATION
}

\author{
M. L. LINGS ${ }^{\mathrm{i})}$ and M. S. DIETZ ${ }^{\mathrm{ii})}$
}

\begin{abstract}
Sand-steel interface tests have been performed using a modified direct shear apparatus that enables accurate dilation measurements to be taken. Average roughness and maximum roughness, when divided by $D_{50}$, are equally good at correlating both friction and dilation data. Below a certain relative roughness threshold, interface behaviour is non-dilatant. Above that threshold, interfaces show classical stress-dilatancy behaviour, with peak values following a simple flow rule. When peak interface friction and dilation angles are normalised by dividing by equivalent direct shear values, dilatant interface test data covering a range of particle sizes, relative densities and surface roughnesses fit a simple linear model when plotted against relative roughness on a logarithmic scale. When relative roughness reaches an upper limit, interface behaviour becomes fully rough, reproducing peak friction and dilation angles in direct shear. Normalised peak interface friction ratios are likely to range between 0.5 and 1.0 for sand on rolled steel surfaces, with the value depending primarily on particle size.
\end{abstract}

Key words: density, dilatancy, direct shear test, friction, grain size, sand, shear strength, steel (IGC: D6)

\section{INTRODUCTION}

Systems in which discrete particles interact with solid surfaces are commonplace, and the shear forces such systems are able to withstand are of practical importance in many fields. There is general agreement that system response is governed by the properties and behaviour of a narrow band of material between the bulk of the granular assembly and the bulk of the solid material, known as the interface. Interface characteristics such as: size, shape, mineralogy and crushability of the grains; density of the deposit and stress level; roughness and hardness of the surface; test method; have all at one time or another been found to be influential in determining behaviour. Perhaps as a result of this complexity, there has been a tendency to distil interface response into a single parameter, namely the peak interface friction angle, $\delta_{\mathrm{p}}^{\prime}$.

In classical soil mechanics, it has long been understood that soil strength is closely linked to the state of packing of the particles, and particularly to the changes in volume during shear. Within rupture zones, soil dilates to reach a critical state, where further shear deformation proceeds without any further volume change. The simplest possible description of the relationship between strength and dilation makes use of the saw-tooth analogy, described for example by Bolton (1986). Sliding on the inclined planes of the teeth causes work to be done against the applied vertical load, thereby making the resistance greater than it would be just from sliding friction between the surfaces.
Despite the strong link between strength and dilation, and the universal acceptance of stress-dilatancy concepts, the role of dilation during interface shearing has been neglected. Indeed, until recently, interface dilation has only been discussed in qualitative terms.

The paper reports the results of a fundamental study of sand-steel interface friction, using coarse, medium and fine sands, and different surfaces of widely varying roughness. Relative densities from dense to loose have been investigated, and the full range of normal stresses available with the apparatus has been explored. A modified version of the conventional direct shear apparatus (DSA) has been used, enabling accurate measurements to be made of both friction and dilation during interface and direct shear tests. Emerging from this is a clear picture of the role of dilation in the peak strength of interfaces.

\section{BACKGROUND}

Potyondy (1961) was the first to make a systematic study of interface friction, and gave ratios of $\delta_{p}^{\prime} / \phi_{p}^{\prime}$ for a wide range of soils on various construction materials, although his tests using sand were done without varying the relative density. Butterfield and Andrawes (1972) showed that interface friction depended on the relative density of the sand, with the ratio of friction between loose and dense tests being approximately constant for a range of construction materials. Acar et al. (1982) studied the effect of both density and stress level on interface fric-

i) Senior Lecturer, Department of Civil Engineering, University of Bristol, UK (Martin.Lings@bristol.ac.uk).

ii) Research Associate, ditto.

The manuscript for this paper was received for review on August 24, 2004; approved on September 1, 2005.

Written discussions on this paper should be submitted before July 1, 2006 to the Japanese Geotechnical Society, 4-38-2, Sengoku, Bunkyo-ku, Tokyo 112-0011, Japan. Upon request the closing date may be extended one month. 


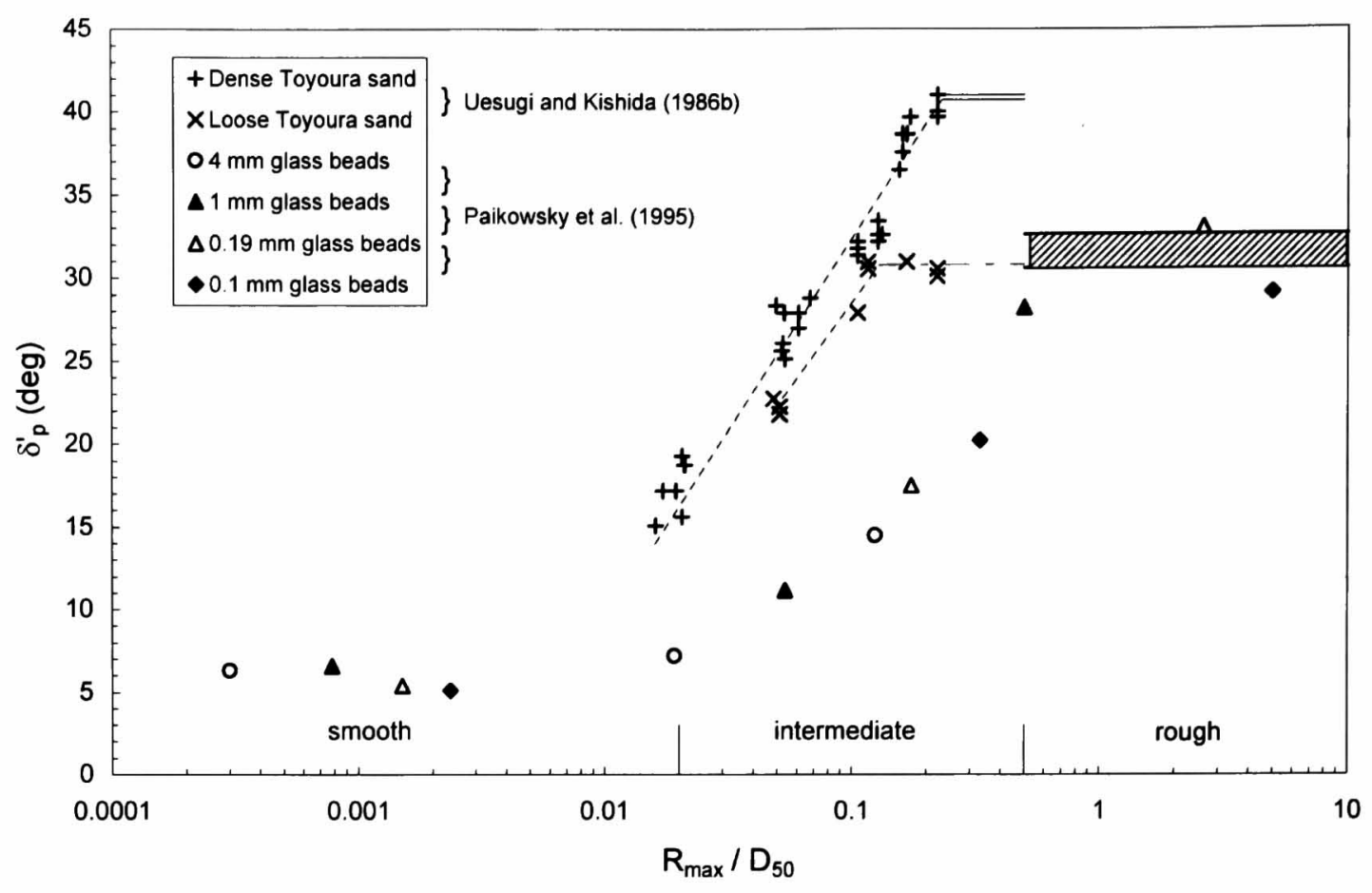

Fig. 1. Peak interface friction vs. normalised roughness

tion. They showed that the ratio $\delta_{\mathrm{p}}^{\prime} / \phi_{\mathrm{p}}^{\prime}$ was independent of relative density, and reduced slightly with increased stress level. Their conclusions were based on only one sand and one surface roughness, neither of which were specified. All three used a DSA, with the sand deposited onto the solid surface, the "type B" configuration identified by Subba Rao et al. (1996).

In contrast, Yoshimi and Kishida (1981) used a ring shear apparatus, with the solid surface lowered onto the sand, the "type A" configuration. They used a range of sands, and surfaces of widely different roughnesses, and were the first to identify the importance of surface roughness. However, because of their "type A" configuration, they were unable to detect changes in interface friction with changes in relative density.

A comprehensive series of tests was reported by Uesugi and Kishida (1986a, 1986b), Kishida and Uesugi (1987) and Uesugi et al. (1988), who used a simple shear apparatus (SSA). This enabled the interface displacement to be separated from the displacement of the sand itself. They investigated a range of sands and surface roughnesses, at various relative densities and stress levels, and introduced the concept of "normalised roughness", which was able to integrate the effects of particle size and surface roughness.

\section{ROUGHNESS}

Roughness is the term used for the smallest-scale surface texture, as distinct from waviness. It can be characterised in a number of ways, but the commonest, in many fields, is the average roughness, $R_{\mathrm{a}}$ (the arithmetic mean absolute deviation of the profile from the centre line). A parameter also used in the geotechnical literature is the maximum roughness, $R_{\max }$ (the height between the highest and lowest points on the profile).

\section{Roughness and Particle Size}

Uesugi and Kishida (1986b) introduced normalised roughness, $R_{\mathrm{n}}$, as a way of accounting for the observation that, in general, a fine sand will mobilise more friction on a given surface than a coarse sand. They defined it as $R_{\max } / D_{50}$, where $R_{\max }$ is obtained using a profile length, $L=D_{50}$. As explained by Kishida and Uesugi (1987), $L=$ $D_{50}$ was adopted instead of $L=2.5 \mathrm{~mm}$, as used originally by Yoshimi and Kishida (1981), to avoid large roughness measurements caused by wavy surfaces. Using normalised roughness, they showed reasonable linear correlations when peak stress ratio was plotted against normalised roughness on a natural scale.

Paikowsky et al. (1995) did interface tests in a dual interface apparatus (DIA), and also used normalised roughness for plotting their data. However, they plotted peak interface friction angle against normalised roughness on a logarithmic scale. Their data for glass beads of various sizes on steel and aluminium surfaces using the DIA are shown in Fig. 1. Their data are seen to fall into three zones, which they termed "smooth", "intermediate" and "rough". The interface friction angle in the rough zone approximates to values obtained in direct shear, shown by the horizontal shaded zone on the right hand side of the figure.

Also shown in Fig. 1 are some data from tests on dense and loose Toyoura sand in the SSA by Uesugi and Kishida (1986b). It can be seen that these data also give reasonable straight lines when plotted on semi- 


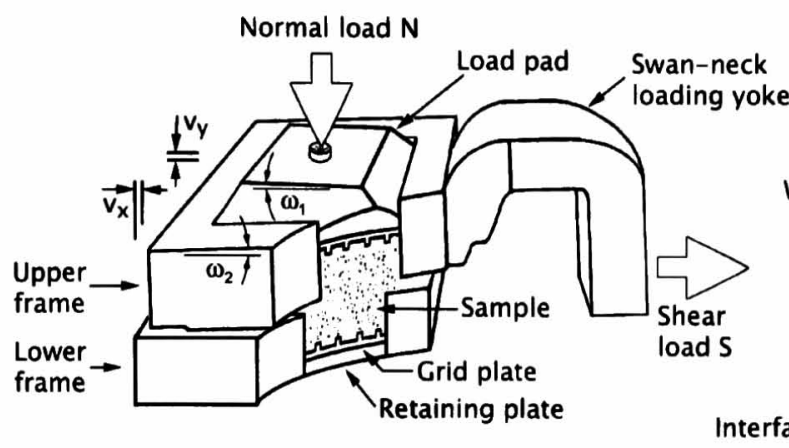

(a)

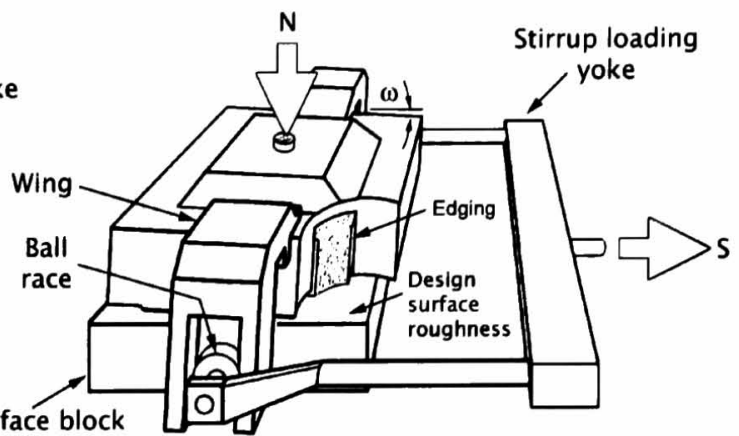

(b)

Fig. 2. (a) Conventional DSA and (b) modified WDSA used for interface testing

logarithmic axes.

Jardine et al. (1993) used average roughness for correlating data, plotting stress ratio against $R_{\mathrm{a}} / D_{50}$ on a natural scale. Subba Rao et al. (1998) adopted the term "relative roughness", $R$, defined as $R_{\mathrm{a}} / D_{\mathrm{av}}$, where $D_{\mathrm{av}}$ is the weighted average particle size of the sand. They presented semi-logarithmic plots of normalised interface friction angle $\left(\delta^{\prime} / \phi^{\prime}\right)$ against relative roughness for a range of sand sizes, and showed a smooth S-shaped curve could be fitted to the data.

\section{Dilation}

Yoshimi and Kishida (1981) were the first to show graphs of the dilation observed in interface tests. Recently, Dove and Jarrett (2002) have focussed on the role of dilation in studying the behaviour of various granular materials against aluminium and geosynthetic surfaces. They describe two limiting types of behaviour: "nondilative" when the interface is smooth, and "dilative" when the interface is rough, mobilising the full strength of the soil. In this paper, the term "non-dilatant" will be used to describe interfaces that produce negligible dilation, and the term "dilatant" will be used to describe any interface that dilates during shear, up to and including those classified as rough.

\section{TESTING}

All the tests described here were carried out using a $100 \mathrm{~mm}$ square DSA, modified to improve the accuracy of the friction and dilation measurements.

\section{Test Apparatus}

The conventional DSA commonly encountered in the UK is shown in Fig. 2(a). The sample is contained within a shearbox, and confined between a retaining plate and load pad, each with its grid plate. The steel shearbox, square on plan, is split horizontally at mid height, forming an upper and lower frame. A normal load $N$ is applied via a load hanger resting on the top of the load pad. The shear load $S$ is applied via a swan neck attached to the upper frame. The shearbox sits in a carriage (not pictured) which, guided by linear bearings, is displaced by a worm drive connected to an electric motor.

The modified apparatus, pictured as used for interface testing, is shown in Fig. 2(b). The swan-neck has been replaced by a pair of "wings" attached to the sides of the upper frame, hence the name winged DSA, abbreviated to WDSA. The shear load is applied at the sample centre through ball races, preventing unwanted forces and moments from acting, and allowing unrestricted dilation. During testing, the load pad is securely fixed to the upper frame, creating the symmetrical arrangement recommended by Jewell and Wroth (1987). This reduces upperframe rotations ( $\omega$ in Fig. 2(b)), which in the conventional DSA are often of different sign between load pad and upper frame ( $\omega_{1}$ and $\omega_{2}$ in Fig. 2(a)). It also simplifies testing, as it removes the intermittent problem of the load pad jamming within the upper frame, and it eliminates the movement of sand vertically past the walls of the shearbox. An initial gap of $5 D_{50}$ is created between the frames before sample deposition, with strips of rubber edging attached to the internal walls to limit sample extrusion. The grid plates are omitted, as they are ineffective at transmitting shear load, and their use leads to dilation being underestimated.

The WDSA retains the simplicity of the conventional DSA, but overcomes its tendency to overestimate friction and underestimate dilation. The modifications to the apparatus and the validation of the results are fully described by Dietz (2000) and Lings and Dietz (2004).

\section{Test Sands}

Three different sands were used in this research. Each was sieved, washed and oven dried before testing and then discarded. The coarse sand was Leighton Buzzard, and because the batch was new, unlike others in the lab, it was termed virgin Leighton Buzzard, VLB. The medium sand, because of its orange or golden colour, was termed medium golden sand, MGS. The fine sand, because of its silver-grey colour, was termed silver fine sand, SFS. A number of tests were performed to characterise the physical properties of these sands, the results of which are given in Table 1 .

Dense and medium dense samples were prepared by pluviation, using a device similar to the multiple sieve 
Table 1. Physical properties of test sands

\begin{tabular}{c|c|c|c|c|c|c|c|c|c}
\hline \multicolumn{1}{c|}{ Sand } & Name & $G_{\mathrm{s}}$ & $e_{\min }$ & $e_{\max }$ & $D_{10}(\mathrm{~mm})$ & $D_{50}(\mathrm{~mm})$ & $D_{60}(\mathrm{~mm})$ & $U_{\mathrm{c}}$ & \multicolumn{1}{c}{ Shape } \\
\hline Coarse & VLB & 2.651 & 0.506 & 0.802 & 0.64 & 0.78 & 0.81 & 1.27 & Rounded \\
\hline Medium & MGS & 2.657 & 0.494 & 0.806 & 0.34 & 0.44 & 0.45 & 1.32 & Sub-rounded \\
\hline Fine & SFS & 2.654 & 0.684 & 1.017 & 0.09 & 0.13 & 0.14 & 1.56 & Sub-rounded \\
\hline
\end{tabular}

Table 2. Formative processes and properties of the surfaces investigated

\begin{tabular}{|c|c|c|c|c|}
\hline Material & Name & Formative process & $R_{\max }(\mu \mathrm{m})$ & $R_{\mathrm{a}}(\mu \mathrm{m})$ \\
\hline Steel & POL & $\begin{array}{l}\text { Abrasion of ground surface using progressively finer grades } \\
\text { of abrasive paper, before polishing with Brasso }\end{array}$ & 1.98 & 0.147 \\
\hline Steel & GND & $\begin{array}{l}\text { Grinding wheel, with direction of striations perpendicular to } \\
\text { direction of shearing }\end{array}$ & 3.85 & 0.356 \\
\hline Steel & ALO & Shot blasting with 120 grit aluminium oxide $\left(D_{50}=0.2 \mathrm{~mm}\right)$ & 24.6 & 2.49 \\
\hline Steel & $\mathrm{SIC}$ & Shot blasting with 16 grit silicone carbide $\left(D_{50}=1.8 \mathrm{~mm}\right)$ & 67.0 & 9.40 \\
\hline Sand & SFS & $\begin{array}{l}\text { Pluviation of SFS onto a uniform coating of Araldite smeared } \\
\text { across the block }\end{array}$ & 202 & 33.7 \\
\hline Sand & MGS & $\begin{array}{l}\text { Pluviation of MGS onto a uniform coating of Araldite smeared } \\
\text { across the block }\end{array}$ & 880 & 114 \\
\hline Sand & VLB & $\begin{array}{l}\text { Pluviation of VLB onto a uniform coating of Araldite smeared } \\
\text { across the block }\end{array}$ & 1320 & 180 \\
\hline
\end{tabular}

pluviator described by Miura and Toki (1982). The rate of deposition was adjusted to alter the density of the deposit by changing the size of the discharge nozzle. Loose samples were prepared by the slow pouring method described by Miura et al. (1997). The top surface of the sample was initially levelled using a small vacuum device (Stroud, 1971), and finally levelled by contact with a greased platen repeatedly lowered onto the surface of the sand to remove excess grains.

\section{Test Surfaces}

As depicted in Fig. 2(b), interface testing was carried out by replacing the lower frame with a series of solid steel blocks, prepared with various roughness magnitudes. The mild steel blocks were machined to have dimensions equivalent to the WDSA's lower frame and had their principal surfaces ground parallel to one another. Four distinct steel roughness magnitudes were investigated, each newly prepared prior to each test using the processes described in Table 2 . Three different sand roughness magnitudes were investigated, formed by fixing each of the three test sands to a steel block as described in Table 2 . These created fully rough surfaces when tested with the same sand, similar to the "perfectly rough" surfaces tested by Butterfield and Andrawes (1972).

\section{Roughness Measurement}

Surface profiles were digitised, recorded and analysed using a Talysurf profilometer, a device that produces a magnified surface profile by dragging a very sensitive stylus along a sample length of surface. It has a spherical tip radius of $2 \mu \mathrm{m}$ and contact force of $0.7-1 \mathrm{mN}$. The nominal vertical resolution of the digitised points was $\pm 0.5 \mathrm{~nm}$, but the resolution of the mechanical elements of the measuring system was probably less. Background noise produced vertical deflections of the stylus tip of around $3 \mathrm{~nm}$.

For the smoother surfaces investigated, roughness parameters were evaluated over a profile length of $5 \mathrm{~mm}$. A total of five profile segments were used to characterise a surface. Each traverse was taken across a different portion of the surface to provide an indication of the uniformity of roughness, which was generally found to be high. For the rougher sand surfaces it was necessary to increase the traverse length to $70 \mathrm{~mm}$ to acquire sufficient profile data to enable an accurate estimate of roughness parameters.

The steeper contact plane between particles and a given surface for fine sands compared to coarse sands has been noted by Uesugi and Kishida (1986b) and Paikowsky et al. (1995), and is the rationale for normalising the measured roughness by the particle size. This difference in contact plane angle is illustrated in Fig. 3 for fine and coarse sand particles superimposed on the ALO surface. As a comparison, the same particles are also shown on the smooth GND surface.

\section{Test Details}

A single LVDT was used to record the carriage displacement, $v_{\mathrm{x}}$, and other LVDTs were used to measure the vertical displacement of the upper frame assembly: Positioned above the front and rear of the apparatus; these instruments recorded average values of vertical 

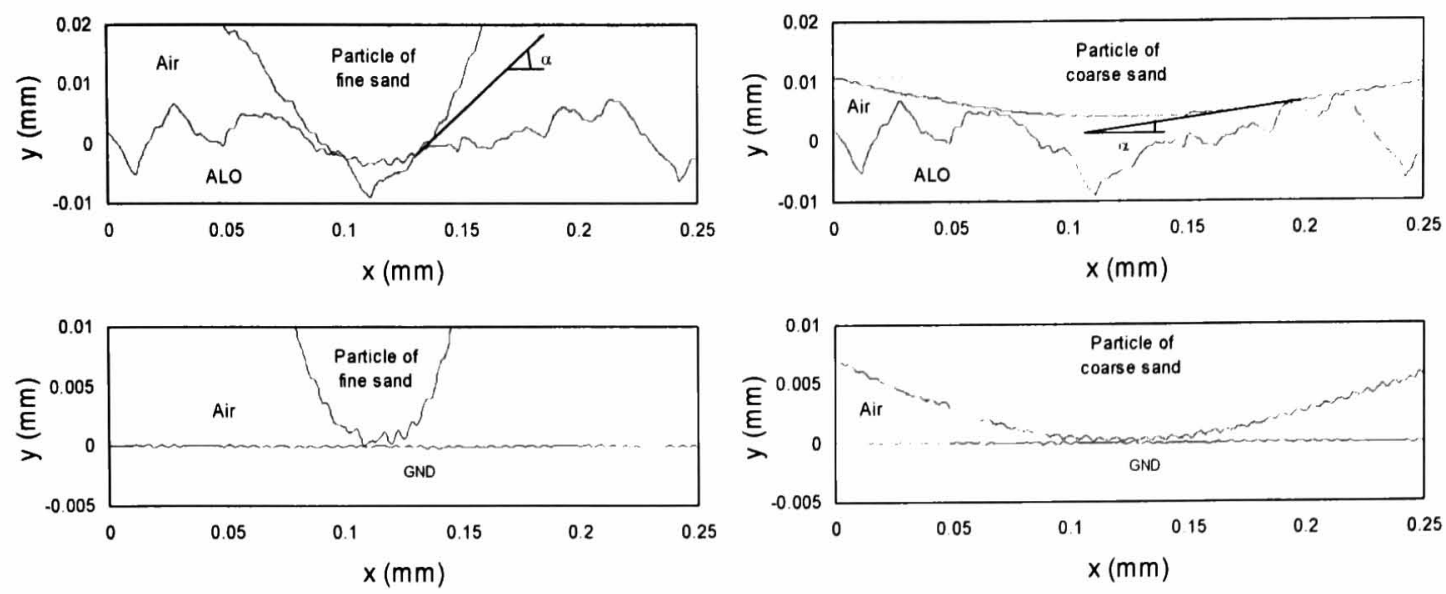

Fig. 3. Profiles of sand grain on steel surface

displacement, $v_{\mathrm{y}}$, as well as the rotation of the upper frame assembly. Clockwise rotations as shown in Fig. 2(b) are taken as positive. All tests were carried out at a constant rate of shear displacement of $1.2 \mathrm{~mm} / \mathrm{min}$.

A number of direct shear tests were performed using the WDSA to provide comparative data. The configuration of these tests was identical to the interface tests, except the interface block was replaced with a conventional lower frame containing the lower half of the sample.

\section{Test Parameters}

In the WDSA, the average stresses obtained from boundary measurements are the vertical normal stress, $\sigma_{y y}^{\prime}$, and the horizontal shear stress $\tau_{\mathrm{yx}}$. Both require knowledge of the plan area of the sample, $A$. It is important to include the self weight of the upper frame assembly and sand, $n$, when deriving $\sigma_{\mathrm{yy}}^{\prime}$ :

$$
\begin{aligned}
\sigma_{\mathrm{yy}}^{\prime} & =\frac{N+n}{A} \\
\tau_{\mathrm{yx}} & =\frac{S}{A}
\end{aligned}
$$

For direct shear tests, the direct shear friction angle, $\phi_{\mathrm{ds}}^{\prime}$, and dilation angle, $\psi$, are obtained from:

$$
\begin{aligned}
& \tan \phi_{\mathrm{ds}}^{\prime}=\tau_{\mathrm{yx}} / \sigma_{\mathrm{yy}}^{\prime} \\
& \tan \psi=d v_{\mathrm{y}} / d v_{\mathrm{x}}
\end{aligned}
$$

For interface tests, the interface friction angle is $\delta^{\prime}$, derived in the same way as $\phi_{\text {ds. }}^{\prime}$. In a similar way, the Greek symbol $\xi(\mathrm{Xi})$ will be used for the interface dilation angle, to distinguish it from $\psi$ (Psi) in a direct shear test.

\section{TEST DATA}

Data from eighteen interface tests, each using a dense sand confined under $25 \mathrm{kPa}$, are presented in Table 3 . High densities and low stress levels were deliberately

\begin{tabular}{|c|c|c|c|c|c|c|}
\hline No. & Sand & Surface & $\begin{array}{c}D_{\mathrm{r}} \\
(\%)\end{array}$ & $\begin{array}{c}\sigma_{y y}^{\prime} \\
(\mathrm{kPa})\end{array}$ & $\begin{array}{c}\delta_{\mathrm{p}}^{\prime} \\
\text { (deg) }\end{array}$ & $\begin{array}{c}\xi_{\mathrm{p}} \\
\text { (deg) }\end{array}$ \\
\hline 1 & VLB & POL & 89 & 25.3 & 11.7 & 0.4 \\
\hline 2 & VLB & GND & 94 & 25.2 & 16.7 & 0.2 \\
\hline 3 & VLB & ALO & 92 & 25.3 & 26.0 & 1.9 \\
\hline 4 & VLB & SIC & 88 & 25.1 & 33.2 & 9.6 \\
\hline 5 & VLB & SFS & 83 & 25.2 & 39.9 & 16.4 \\
\hline 6 & VLB & MGS & 89 & 25.2 & 47.5 & 24.8 \\
\hline 7 & VLB & VLB & 86 & 25.2 & 48.1 & 26.6 \\
\hline 8 & MGS & POL & 94 & 25.2 & 13.7 & 0.4 \\
\hline 9 & MGS & GND & 93 & 25.3 & 15.3 & 0.0 \\
\hline 10 & MGS & ALO & 94 & 25.1 & 27.7 & 3.7 \\
\hline 11 & MGS & SIC & 93 & 25.2 & 39.0 & 14.1 \\
\hline 12 & MGS & SFS & 92 & 25.3 & 47.1 & 24.5 \\
\hline 13 & MGS & MGS & 93 & 25.3 & 49.0 & 26.3 \\
\hline 14 & SFS & POL & 99 & 25.3 & 10.9 & 0.4 \\
\hline 15 & SFS & GND & 96 & 25.3 & 15.3 & 0.1 \\
\hline 16 & SFS & ALO & 94 & 25.2 & 36.5 & 10.2 \\
\hline 17 & SFS & SIC & 92 & 25.3 & 42.8 & 15.2 \\
\hline 18 & SFS & SFS & 97 & 25.2 & 43.2 & 17.0 \\
\hline
\end{tabular}
chosen to maximise the amount of dilation. Data from equivalent direct shear tests at similar density and stress
Table 3. Dense interface tests at $25 \mathrm{kPa}$

level are presented in Table 4. Stress ratio, vertical displacement, rate of dilation and upper frame rotation are plotted against horizontal displacement in Fig. 4 for the three test sands to illustrate the main aspects of behaviour.

Generally, increases in roughness push each set of lines upwards towards those recorded in direct shear. It can be seen that the maximum stress ratios and dilation rates recorded on the fully rough surfaces agree well with the direct shear data, although the rate of strain softening is faster, and the overall dilation smaller, than equivalent 
direct shear tests. Also upper frame rotations for fully rough surfaces are generally higher than their direct shear counterparts.

A peak state can be identified in all tests, and associated parameters are indicated in tables and figures with the subscript $p$. Post peak, rates of dilation soon fall to zero as volume changes cease and a relatively steady stress ratio is mobilised. In fact, it is only the upper-frame

Table 4. Direct shear tests

\begin{tabular}{c|l|c|r|r|r}
\hline No. & Sand & $\begin{array}{c}D_{\mathrm{r}} \\
(\%)\end{array}$ & $\begin{array}{c}\sigma_{\mathrm{yy}}^{\prime} \\
(\mathrm{kPa})\end{array}$ & $\begin{array}{c}\left(\phi_{\mathrm{ds}}^{\prime}\right)_{\mathrm{p}} \\
(\mathrm{deg})\end{array}$ & $\begin{array}{c}\psi_{\mathrm{p}} \\
(\mathrm{deg})\end{array}$ \\
\hline 1 & VLB & 97 & 25.4 & 48.2 & 24.9 \\
\hline 2 & MGS & 99 & 25.4 & 49.0 & 27.4 \\
\hline 3 & SFS & 92 & 25.2 & 42.1 & 17.2 \\
\hline 4 & MGS & 73 & 25.3 & 43.6 & 18.4 \\
\hline 5 & MGS & 64 & 25.4 & 40.9 & 15.4 \\
\hline 6 & MGS & 20 & 25.5 & 31.9 & 1.3 \\
\hline 7 & VLB & 90 & 88.0 & 46.1 & 22.9 \\
\hline 8 & VLB & 90 & 169.7 & 45.5 & 22.0 \\
\hline 9 & VLB & 91 & 251.3 & 45.1 & 20.8 \\
\hline
\end{tabular}
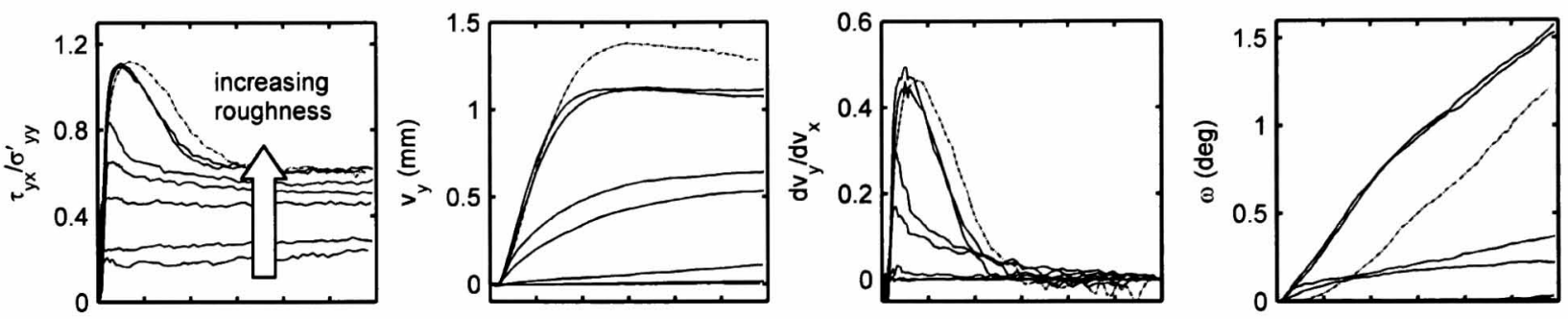

(a)
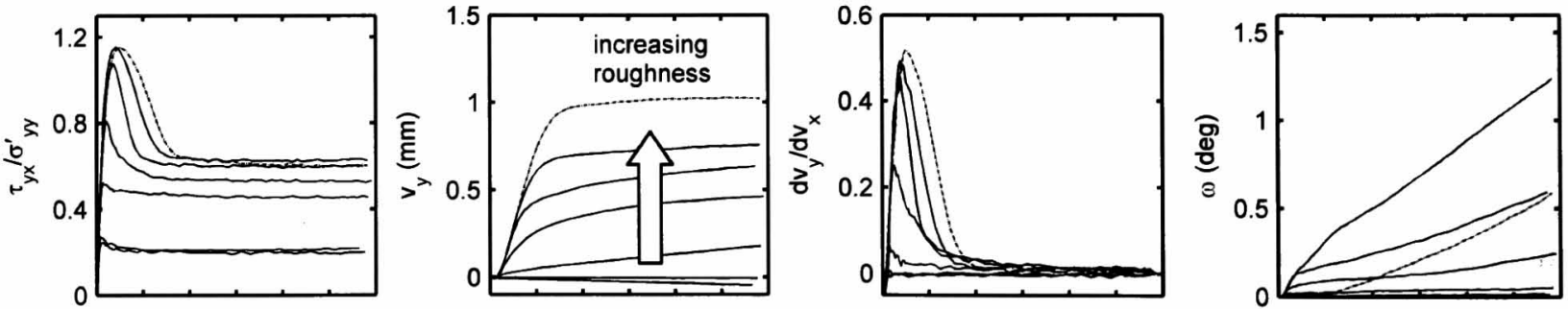

(b)
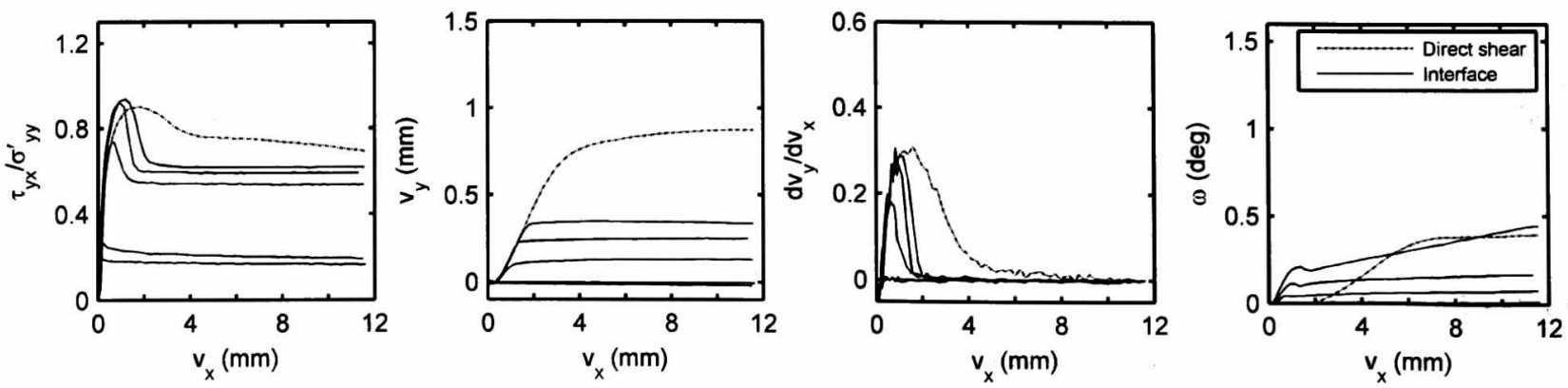

(c)

Table 5. Additional medium sand interface tests at various densities

\begin{tabular}{c|c|c|c|c|c|c}
\hline No. & Sand & Surface & $\begin{array}{c}D_{\mathrm{r}} \\
(\%)\end{array}$ & $\begin{array}{c}\sigma_{\mathrm{yy}}^{\prime} \\
(\mathrm{kPa})\end{array}$ & $\begin{array}{c}\delta_{\mathrm{p}}^{\prime} \\
(\mathrm{deg})\end{array}$ & $\begin{array}{c}\xi_{\mathrm{p}} \\
(\mathrm{deg})\end{array}$ \\
\hline 1 & MGS & GND & 78 & 25.1 & 13.3 & 0.1 \\
\hline 3 & MGS & GND & 69 & 25.2 & 11.5 & 0.1 \\
\hline 4 & MGS & GND & 23 & 25.1 & 10.8 & 0.1 \\
\hline 5 & MGS & ALO & 69 & 25.3 & 25.1 & 2.3 \\
\hline 6 & MGS & ALO & 24 & 25.3 & 22.6 & 0.0 \\
\hline 7 & MGS & SIC & 75 & 25.3 & 35.8 & 12.7 \\
\hline 8 & MGS & SIC & 68 & 25.4 & 33.4 & 11.2 \\
\hline 9 & MGS & SIC & 22 & 25.1 & 29.1 & 1.5 \\
\hline 10 & MGS & SFS & 70 & 25.2 & 42.8 & 18.2 \\
\hline 11 & MGS & SFS & 26 & 25.3 & 33.7 & 4.2 \\
\hline 12 & MGS & MGS & 78 & 25.3 & 43.4 & 18.6 \\
\hline 13 & MGS & MGS & 62 & 25.4 & 40.6 & 14.6 \\
\hline 14 & MGS & MGS & 27 & 25.2 & 33.9 & 3.6 \\
\hline
\end{tabular}


rotations with the rougher surfaces that proceed with any vigour.

Additional tests were performed to investigate the effect of sand density on interface response. These tests were carried out with MGS, and test data are presented in Table 5. Another series of tests investigated the effect of confining stress on interface response. These tests were carried out with VLB, and test data are presented in Table 6.

\section{CORRELATION OF RESULTS}

The normalised roughness of Uesugi and Kishida (1986b) requires a large number of $R_{\max }$ measurements, each derived for a short profile segment of length $D_{50}$. Different sands require different profile lengths and the resulting average values may be different for each test sand. Here, profiles of a much greater length have been used, so the $R_{\max }$ values are not equivalent, and cannot be used to derive the parameter $R_{\mathrm{n}}$. Nevertheless, values of $R_{\max } / D_{50}$ have been calculated for correlation purposes,
Table 6. Additional coarse sand interface tests at various stress-levels

\begin{tabular}{c|c|c|c|c|c|c}
\hline No. & Sand & Surface & $\begin{array}{c}D_{\mathrm{r}} \\
(\%)\end{array}$ & $\begin{array}{c}\sigma_{y y}^{\prime} \\
(\mathrm{kPa})\end{array}$ & $\begin{array}{c}\delta_{\mathrm{p}}^{\prime} \\
(\mathrm{deg})\end{array}$ & $\begin{array}{c}\xi_{\mathrm{p}} \\
(\mathrm{deg})\end{array}$ \\
\hline 1 & VLB & POL & 99 & 128.0 & 14.6 & 0.6 \\
\hline 2 & VLB & POL & 93 & 251.1 & 16.1 & 0.9 \\
\hline 3 & VLB & ALO & 93 & 25.2 & 29.1 & 5.2 \\
\hline 4 & VLB & ALO & 95 & 86.9 & 26.1 & 1.2 \\
\hline 5 & VLB & ALO & 99 & 169.4 & 25.1 & 1.9 \\
\hline 7 & VLB & ALO & 91 & 251.0 & 26.1 & 2.9 \\
\hline 8 & VLB & SIC & 90 & 25.1 & 31.4 & 8.6 \\
\hline 9 & VLB & SIC & 99 & 128.6 & 29.2 & 4.5 \\
\hline 10 & VLB & VLB & 70 & 86.9 & 45.6 & 22.1 \\
\hline 11 & VLB & VLB & 74 & 169.6 & 45.4 & 21.6 \\
\hline 12 & VLB & VLB & 74 & 251.6 & 44.7 & 21.2 \\
\hline
\end{tabular}

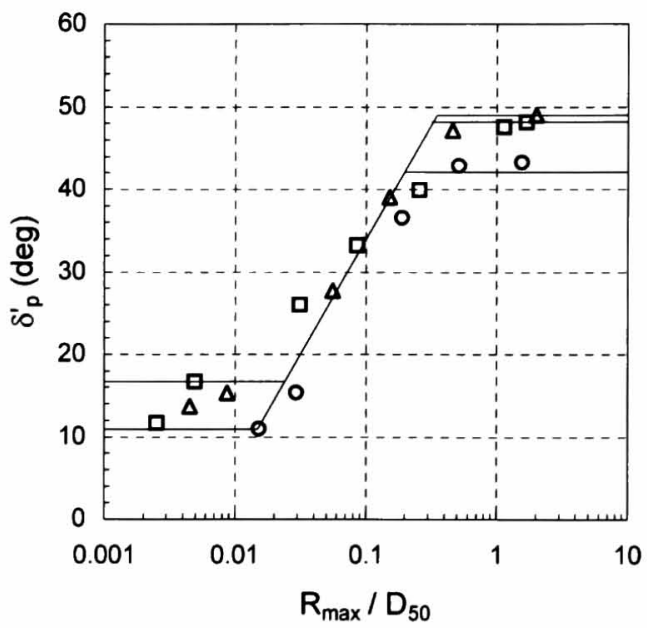

(b)

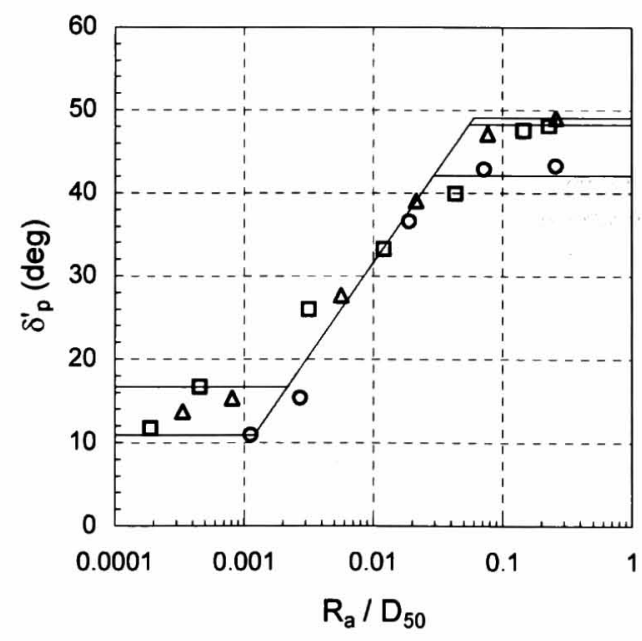

(d)

Fig. 5. Effect of particle size on interface friction angles for dense sands at $25 \mathrm{kPa}$ 


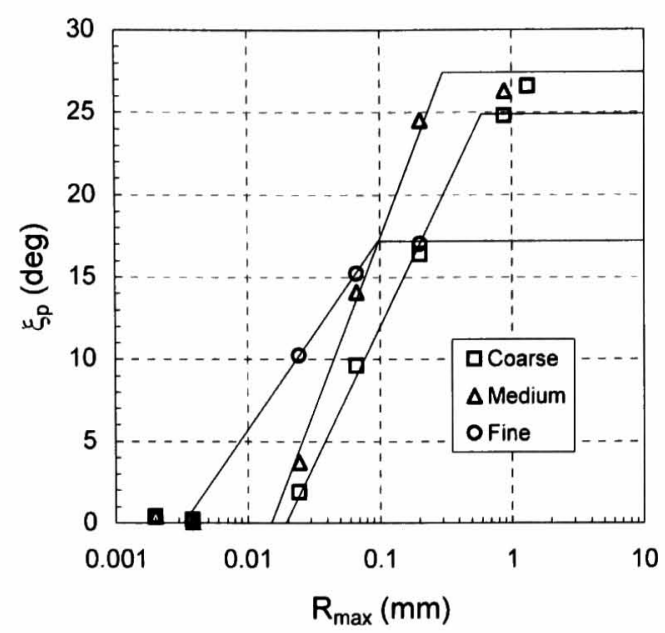

(a)

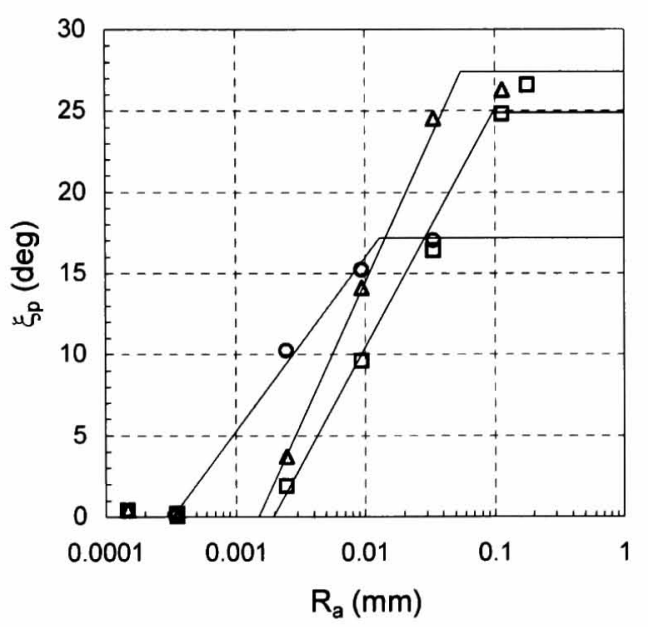

(c)

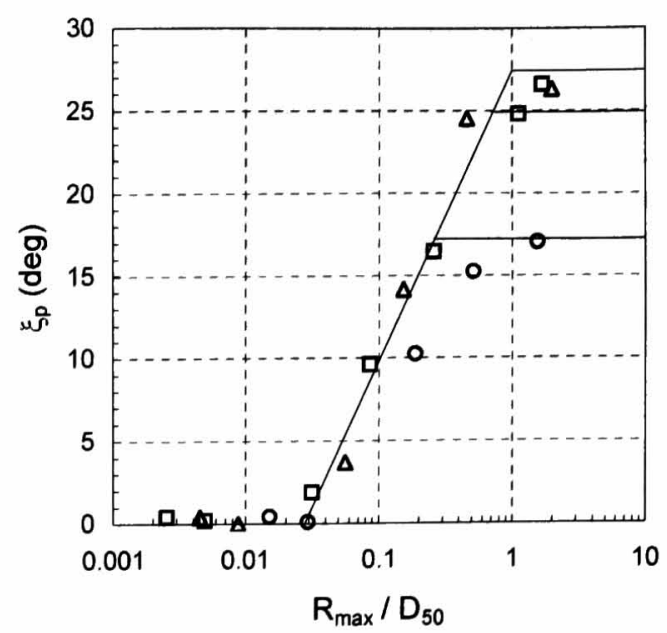

(b)

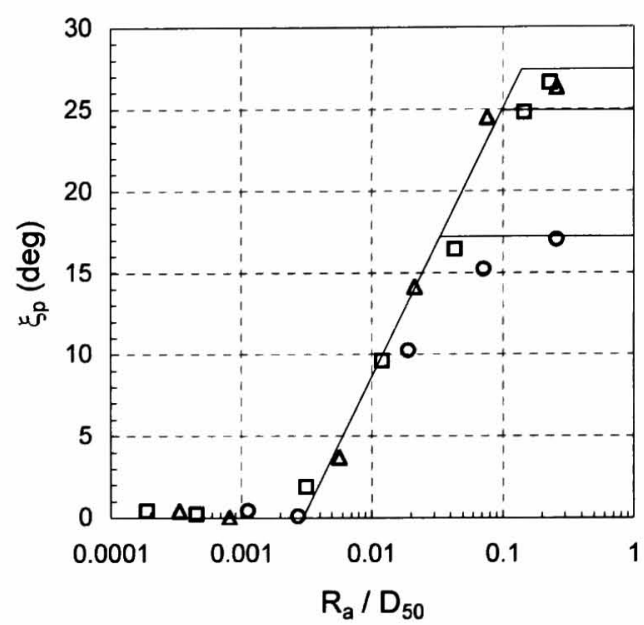

(d)

Fig. 6. Effect of particle size on interface dilation angles for dense sands at $25 \mathrm{kPa}$

but it should be noted that they are not $R_{\mathrm{n}}$ values.

$R_{\mathrm{a}}$ values have been derived, from which values of relative roughness $\left(R_{\mathrm{a}} / D_{50}\right)$ have been calculated. These are not strictly the same as that defined by Subba Rao et al. (1998), although because $D_{50} \approx D_{\mathrm{av}}$, the difference is very small.

Because of the way the interface blocks were prepared, they do not possess any significant waviness component, and it will be shown that $R_{\max }$ and $R_{\mathrm{a}}$ are equally good at correlating results. Values of both $R_{\max }$ and $R_{\mathrm{a}}$ are given in Table 2.

\section{Effect of Particle Size}

Figure 5(a) plots interface friction angles from Table 3 (dense sands at $25 \mathrm{kPa}$ ) against $R_{\max }$. Logarithmic scales for roughness have been used throughout, as this mirrors the way particle sizes are normally plotted, and permits the easy presentation of low roughness values. Best-fit lines added to the data show a reasonable linear increase in $\delta_{p}^{\prime}$ with increasing roughness for each sand. Horizontal lines at high roughness values correspond to the $\left(\phi_{\mathrm{ds}}^{\prime}\right)_{\mathrm{p}}$ values given in Table 4 .

Figure 5(b) plots the same data, but against $R_{\max } / D_{50}$. The data in the intermediate zone now show a reasonably unique linear relationship with normalised roughness. The data in the smooth zone lie within a narrow band, and show a less pronounced reduction in $\delta_{\mathrm{p}}^{\prime}$ with reducing normalised roughness.

Figures 5(c) and (d) present the same data, but using $R_{\mathrm{a}}$ instead of $R_{\max }$. Exactly the same patterns of behaviour are observed, and relative roughness shows the same reasonably unique linear correlation. Thus Paikowsky et al.'s (1995) framework is not restricted to normalised roughness, but works equally well with relative roughness, $R_{\mathrm{a}} / D_{50}$. All subsequent plots will use $R_{\mathrm{a}}$.

In Fig. 6, interface dilation angles, $\xi_{\mathrm{p}}$, are plotted in exactly the same way as the $\delta_{\mathrm{p}}^{\prime}$ values. The horizontal upper bounds at large roughness values are the $\psi_{\mathrm{p}}$ values from Table 4, and like the friction data, show good agreement between fully rough interface and direct shear tests. The lower bound is the zero dilation line. When normalised by $D_{50}$, all the data again show a reasonably 

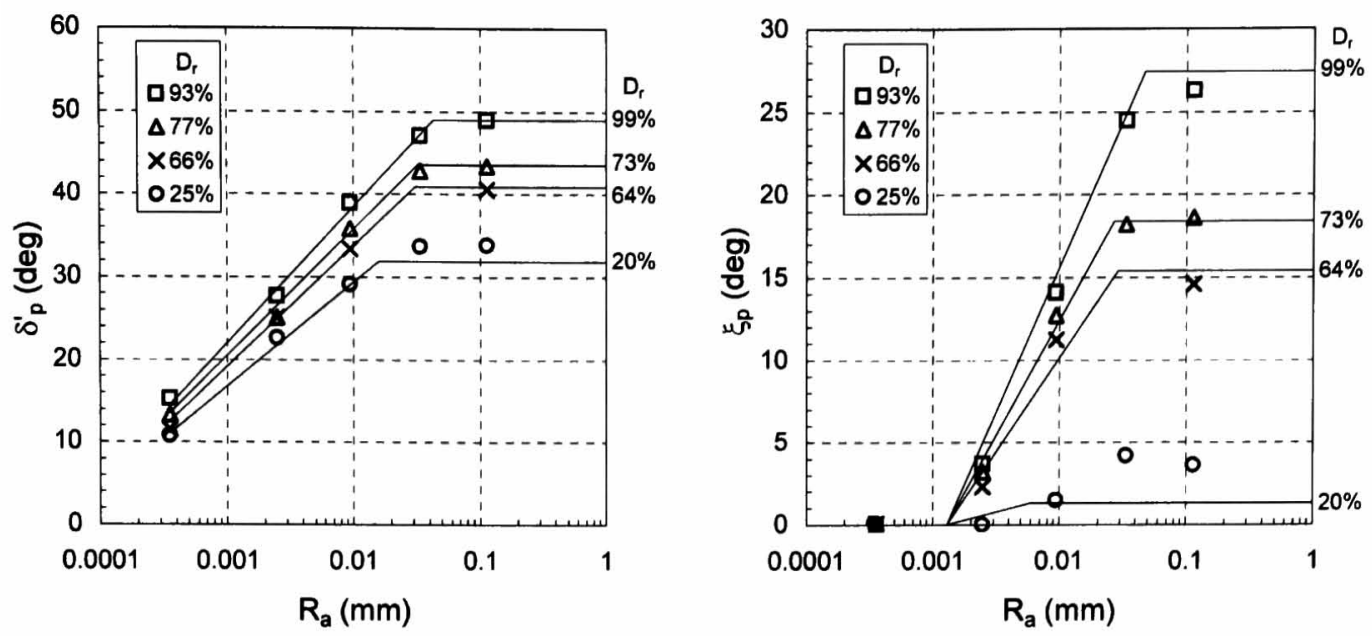

(a)
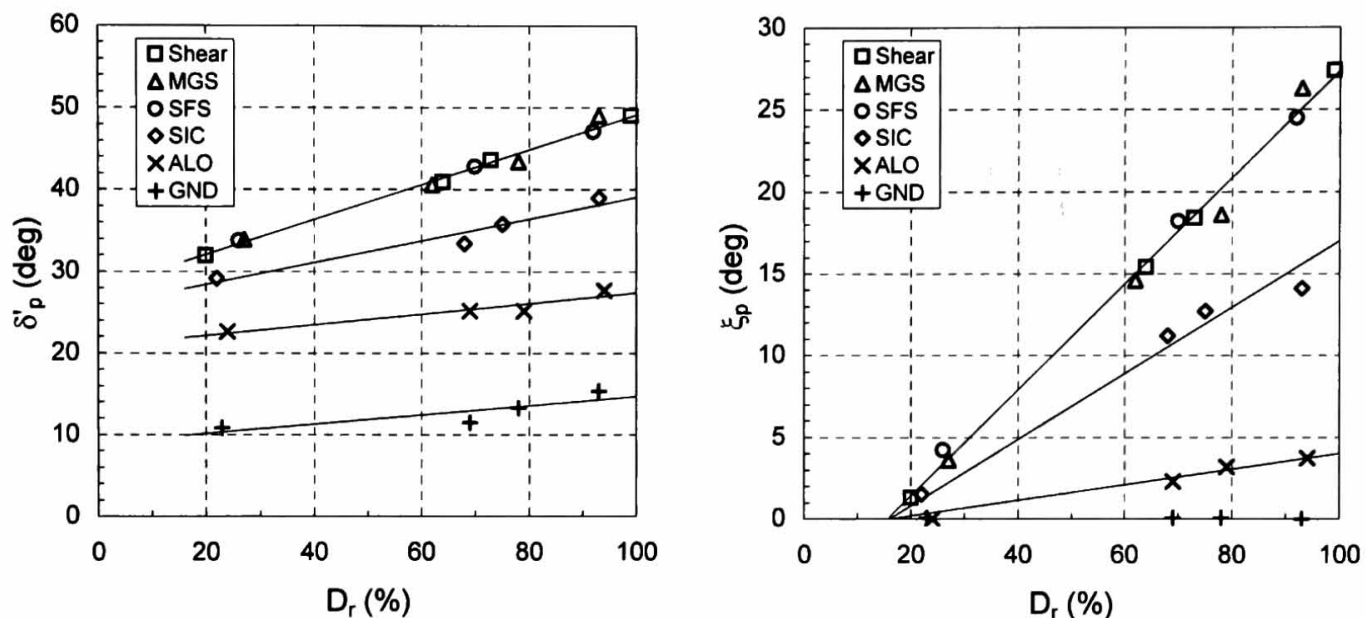

(b)

Fig. 7. Effect of density on interface friction and dilation angles for medium sand under $25 \mathrm{kPa}$ : (a) using roughness axes and (b) using relative density axes

unique relationship. Thus Paikowsky et al.'s (1995) framework, formulated using peak stress data, works equally well with peak dilation data, and is again independent of the roughness parameters adopted.

It will be shown later that the fit of the data, especially the dilation, can be further improved by normalising the friction and dilation axes.

\section{Effect of Density}

Figure 7(a) plots interface friction and dilation angles at various densities from Table 5 (MGS at $25 \mathrm{kPa}$ ) against $R_{\mathrm{a}}$. Because a single test sand was used, roughness has been used rather than relative roughness. As before, horizontal lines at high roughness values correspond to the $\left(\phi_{\mathrm{ds}}^{\prime}\right)_{\mathrm{p}}$ and $\psi_{\mathrm{p}}$ values given in Table 4 . The relative densities given within the figure are averages for the dilatant interface tests; values at the edge are for direct shear. Mismatches between interface and direct shear data at high roughness values are mainly due to differences in relative density. There is a reasonably linear increase in $\delta_{\mathrm{p}}^{\prime}$ and $\xi_{\mathrm{p}}$ with increasing roughness for each density. In general, decreasing the density pushes the lines downwards.

Figure 7(b) plots the same data against relative density. Best-fit lines added to the data show a clear linear increase in $\delta_{\mathrm{p}}^{\prime}$ and $\xi_{\mathrm{p}}$ with increasing relative density for each roughness.

According to Uesugi and Kishida (1986b), density only affects the upper limit of strength. Similarly, Paikowsky et al. (1995) claimed that density only affects strength in the rough zone; they also stated that dilatancy practically does not exist in the intermediate zone. But here it is seen that density has a clear influence on behaviour across the whole of the intermediate zone, in terms of both friction and dilation.

\section{Effect of Stress Level}

Figure 8 plots interface friction and dilation angles at various stress levels from Table 6 (dense VLB) against $R_{\mathrm{a}}$. There is a general trend for both friction and dilation 

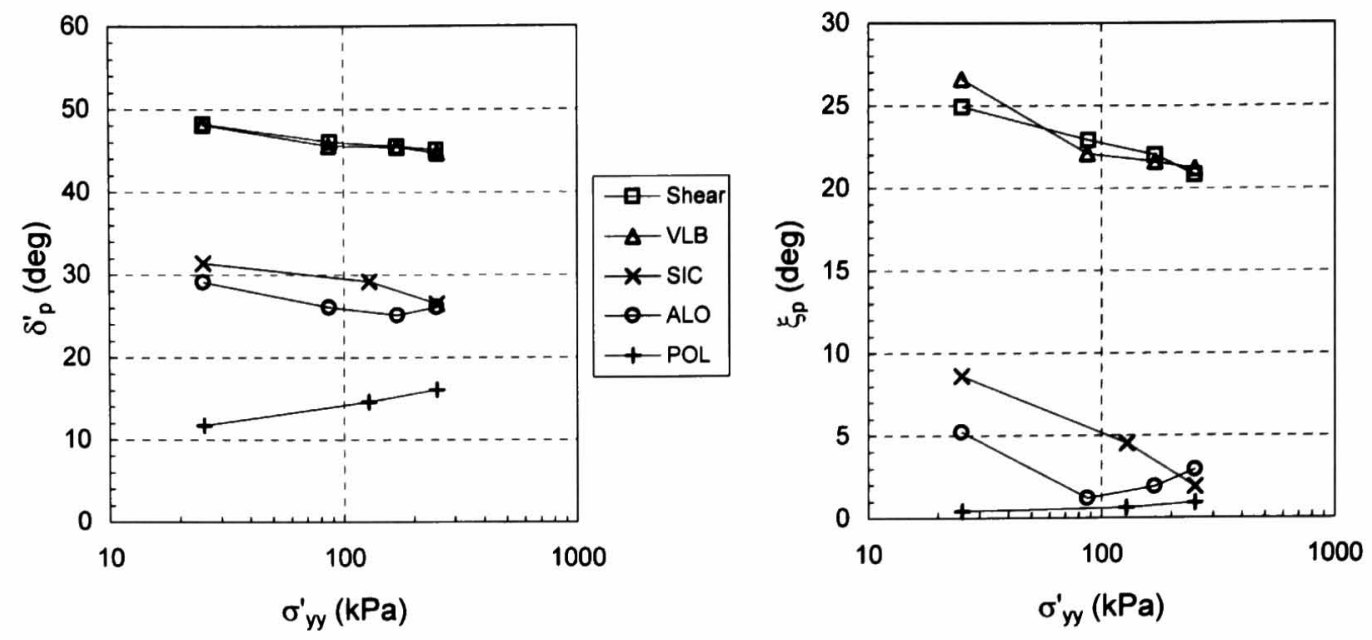

Fig. 8. Effect of normal stress on interface friction and dilation angles for dense coarse sand

angles to reduce with increasing normal stress. The exception to this is when the surface is very smooth, when there is a clear increase in friction angle with increasing normal stress. This is thought to be due to an increase in the amount of ploughing at higher stresses.

\section{STRESS-DILATANCY}

Peak friction and dilation angles for all 44 interface tests from Tables 3, 5 and 6 are plotted against each other in Fig. 9. The data fall into two distinct groups: those with negligible dilation, and those with clear dilation that show classical stress-dilatancy behaviour. The transitional value of $\delta_{\mathrm{p}}^{\prime}$ between the two is approximately $25^{\circ}$, and the dilatant responses correlate well with a flow rule that echoes the simple saw-tooth model of dilation:

$$
\delta_{\mathrm{p}}^{\prime}=25^{\circ}+\xi_{\mathrm{p}}
$$

The sand-on-steel interface tests are shown with open symbols, and they fit this flow rule within the error bounds shown of $\pm 3^{\circ}$. The sand-on-sand interface tests are shown with solid symbols, and they appear to follow a trend with a larger intercept and smaller slope, perhaps echoing Bolton's (1986) flow rule, where only part of the dilation is converted into additional strength. It may be that there is a slight difference between sand-on-sand, and sand-on-steel, interface behaviour.

This clear separation between non-dilatant and dilatant responses suggests that there are two distinct mechanisms by which increases in surface roughness bring about increases in peak strength. The first is associated with smooth surfaces, where the motion of the particles is characterised by sliding at the contact with the surface (Uesugi et al., 1988). Increases in roughness then merely increase the size of asperities that particles need to plough through for interface displacement to occur. The second is associated with intermediate and rough surfaces, where the motion of the particles is increasingly characterised by rolling, resulting in dilation. Increases in roughness, and increases in density, bring about increased dilation

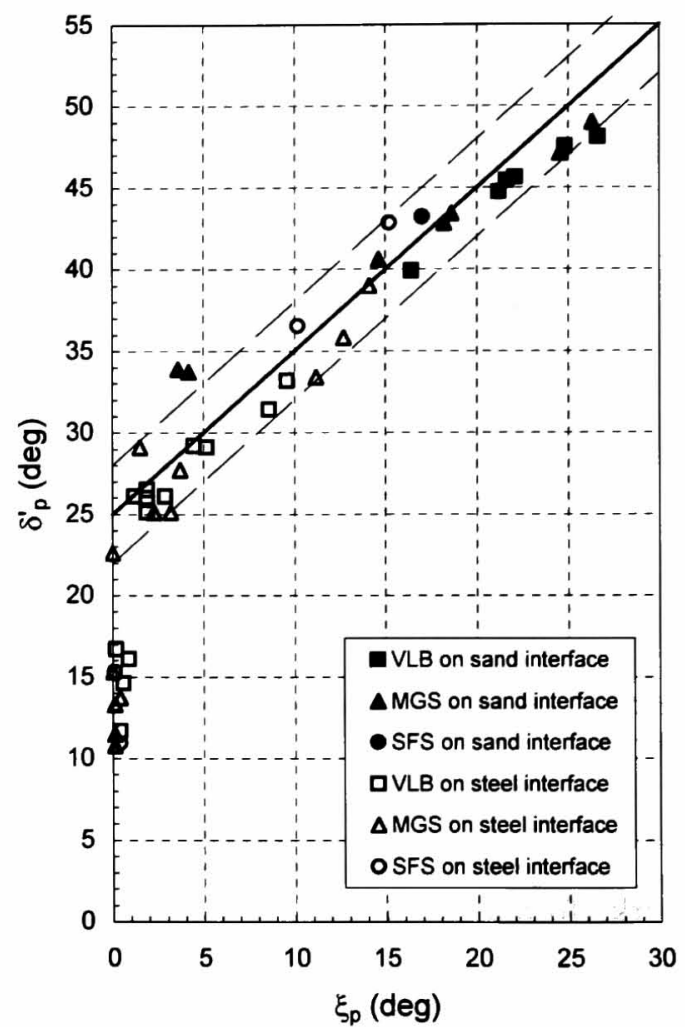

Fig. 9. Peak stress-dilatancy response of interfaces

and a resulting increase in strength.

\section{Normalised Plots}

In the light of the clear stress-dilatancy behaviour shown by interfaces, one would expect to find a clear linkage between friction and dilation elsewhere in the data, particularly the relative roughness thresholds that mark changes in behaviour. When comparing Figs. 5 and 6 , it is evident that this is not the case with the trend lines drawn, particularly the relative roughness at which behaviour becomes fully rough.

Because of the large spread of maximum dilation an- 

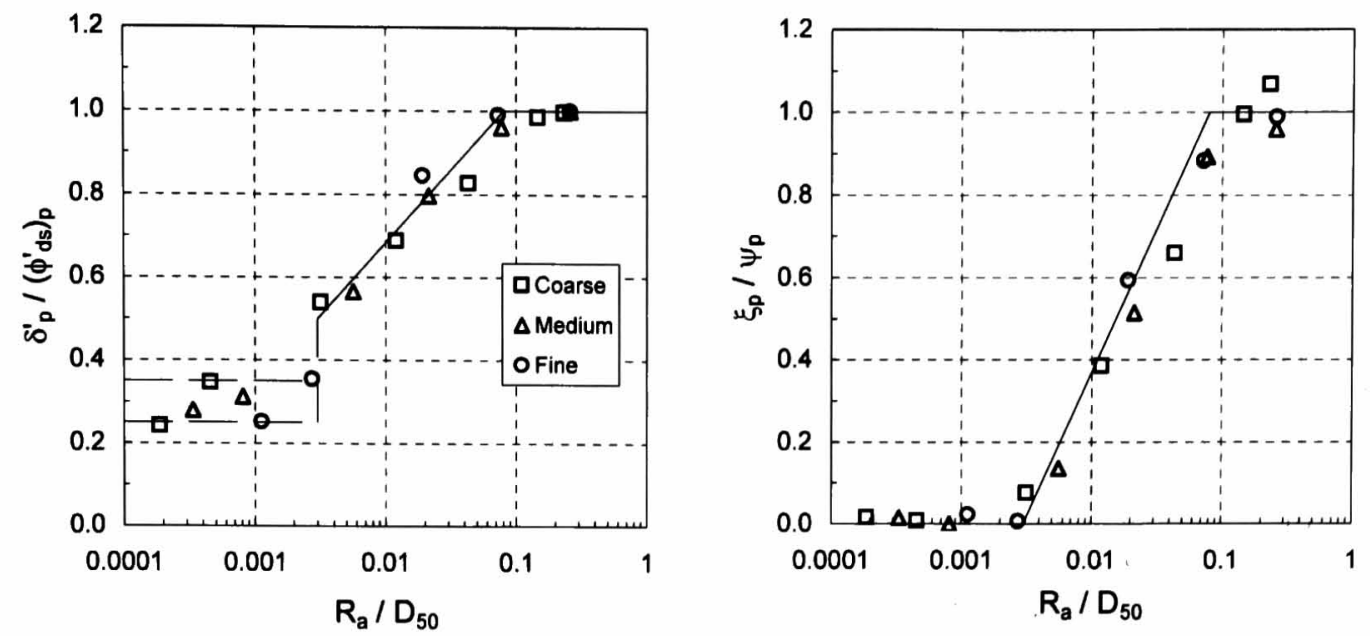

Fig. 10. Normalised interface friction and dilation vs. relative roughness (dense sands at $25 \mathrm{kPa}$ )

gles between the three test sands, the friction and dilation data have been normalised in Fig. 10 by dividing by the maximum direct shear values in Table 4. An exception has been made with SFS, where the fully rough interface friction value has been used instead, because of evidence that the value measured in direct shear is too low (see Fig. 4(c)), thought to be due to the formation of oblique rupture planes.

Some explanation is required as to how the trend lines in Fig. 10 have been deduced. There is a clear jump in friction values, and the onset of dilation, at $R_{\mathrm{a}} /$ $D_{s 0} \approx 0.003$. This value is taken as the boundary between the smooth and intermediate zones. From Fig. 7(b) it is seen that medium sand on SFS produces fully rough behaviour, therefore the boundary between the intermediate and rough zones has been taken at $R_{\mathrm{a}} / D_{50} \approx 0.08$. The onset of dilation occurs with a friction angle of around $25^{\circ}$ (Fig. 9), and the maximum direct shear friction angle measured is $49^{\circ}$ (MGS). This gives a normalised friction value at the onset of dilation of 25 / $49 \approx 0.5$. Use of these values successfully integrates the normalised friction and dilation data for dilatant interfaces in the light of the flow rule.

In the smooth zone, dilation is negligible, and normalised friction values lie within a band between 0.25 and 0.35 . It is not clear what the overall trend of the data is, as emphasised by the dotted lines, except that relative roughness is no longer able to bring the data together onto a single line. A logarithmic scale expands the graph at low relative roughness, so the normalised friction data have been plotted on a natural scale in Fig. 11 to put this zone into perspective.

\section{Relative Density}

For fully rough interfaces, friction and dilation data both lie on clear straight lines when plotted against relative density (Fig. 7(b)), in spite of having a spread of relative densities about the nominal value. In order to normalise data correctly from Fig. 7(a), the calculation has been done using interpolated values from the fully-

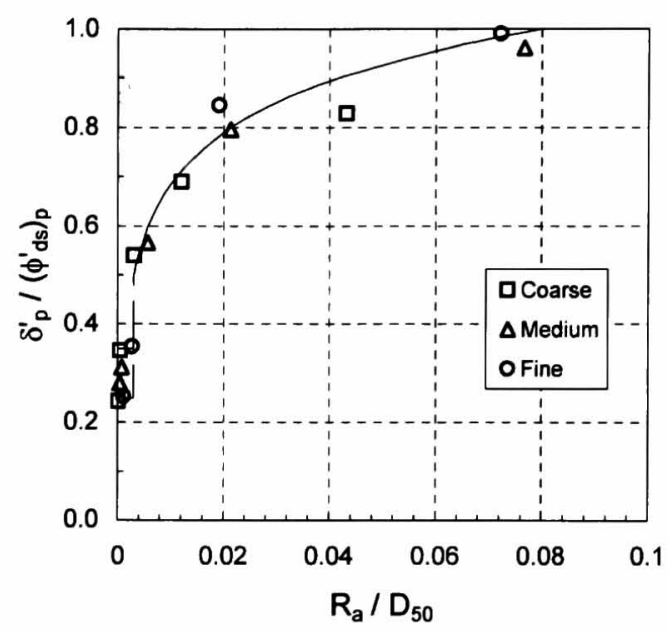

Fig. 11. Normalised interface friction vs. relative roughness (natural scale)

rough best-fit lines appropriate to the relative density of each interface test.

The best-fit regression line through the fully-rough dilation data gives maximum $\xi_{\mathrm{p}}=27.5^{\circ}$, and zero dilation at $D_{\mathrm{r}}=16 \%$. This latter value is smaller than shown in Bolton's (1986) Fig. 7, where he shows a similar plot, with zero dilation at $D_{\mathrm{r}}=23 \%$. But the data shown in that plot trend to a lower value, and the stress level is higher, thus the value of $16 \%$ is considered entirely reasonable.

The best-fit line through the fully-rough friction data gives $\delta_{\mathrm{p}}^{\prime}=49^{\circ}$ at $D_{\mathrm{r}}=100 \%$, and $\delta_{\mathrm{p}}^{\prime}=31^{\circ}$ at $D_{\mathrm{r}}=16 \%$. This lower value represents the critical state, and is in good agreement with measured large displacement values for MGS of $\left(\phi_{\mathrm{ds}}^{\prime}\right)_{\mathrm{ld}}$ between $31^{\circ}$ and $32^{\circ}$.

Normalised friction and dilation data for medium sand at $25 \mathrm{kPa}$ are shown in Fig. 12. Threshold average roughness values of $0.0013 \mathrm{~mm}$ and $0.035 \mathrm{~mm}$ have been used, which are equivalent to those in Fig. 10 when $D_{50}=0.44$ $\mathrm{mm}$. It can be seen that normalisation brings all the data together, except for some loose tests that show scatter. 

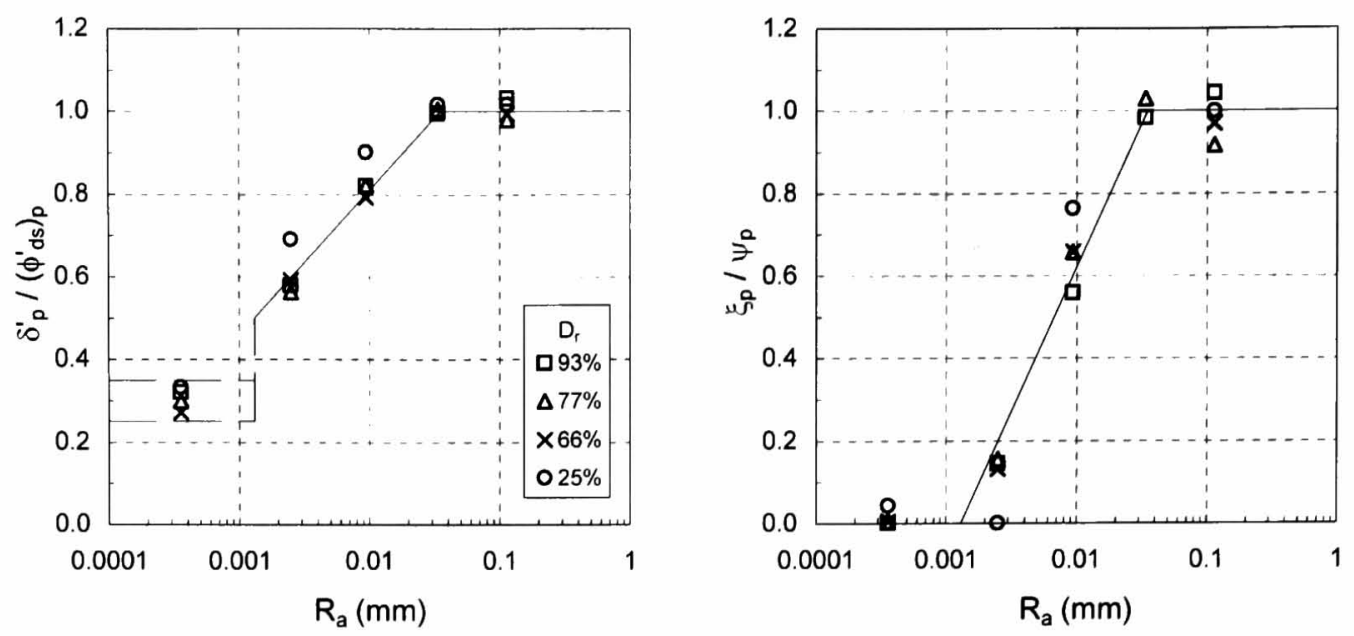

Fig. 12. Normalised interface friction and dilation vs. roughness (medium sand at $25 \mathrm{kPa}$ )
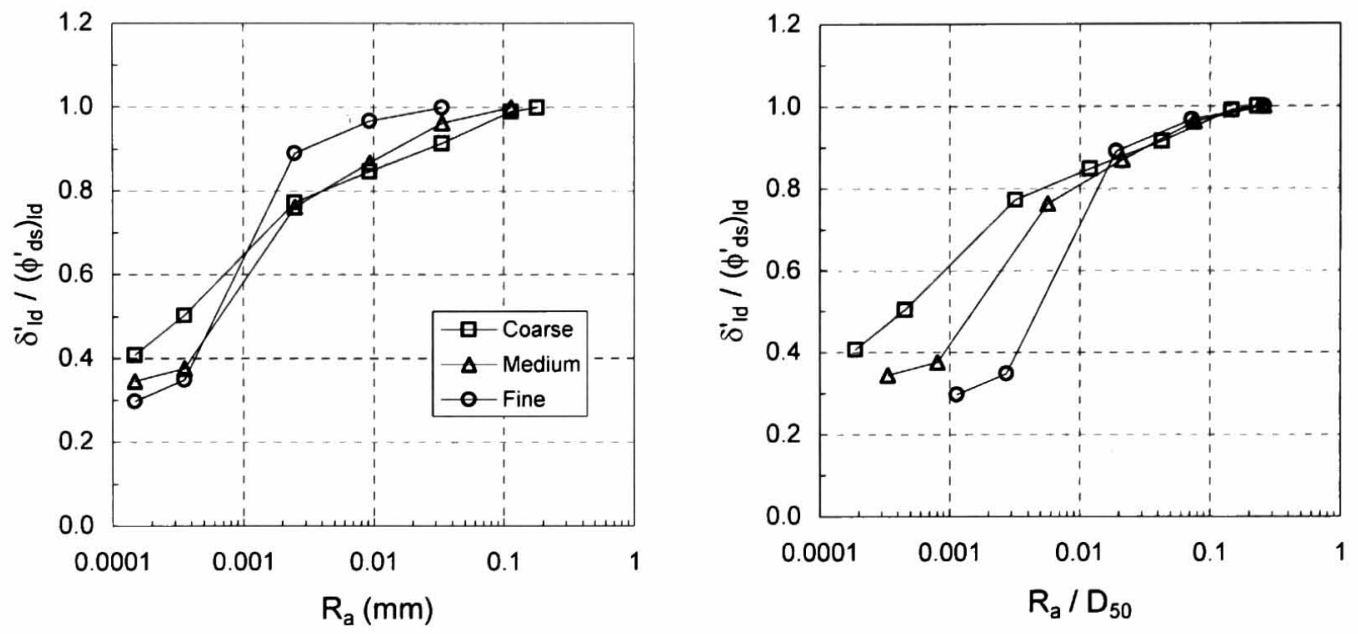

Fig. 13. Large displacement interface friction vs. roughness (densely-deposited sands at $25 \mathrm{kPa}$ )

\section{Large Displacement}

Whilst not the focus of this paper, it is helpful to look briefly at large displacement data obtained from the end of interface tests, and the results from the dense tests at $25 \mathrm{kPa}$ are plotted in Fig. 13. Although $\left(\phi_{\mathrm{ds}}^{\prime}\right)_{\mathrm{ld}}$ values are similar for the three sands, the data have been normalised as before. Roughness and relative roughness have both been plotted, and the latter is successful in integrating the large displacement data, but only for dilatant tests. The non-dilatant tests show entirely different trends, suggesting there may be different mechanisms at work. This has similarities with the non-dilatant peak friction data shown in Fig. 10.

\section{DISCUSSION}

All interface tests at $25 \mathrm{kPa}$ are combined in Fig. 14. Apart from some loose tests where there is scatter, use of normalisation and relative roughness unifies the data, irrespective of particle size or sample density. This is true for both friction and dilation.

The tests reported here used sands with rounded/sub- rounded grains. Subsequent testing with angular sands may reveal more scatter. Further testing is also required around the $R_{\mathrm{a}} / D_{50} \approx 0.003$ threshold to clarify the transition between non-dilatant and dilatant behaviour, shown here simply as a vertical line on the friction diagram.

When the roughness of rolled steel is combined with the standard particle-size limits for sands, the resulting range of relative roughness values matches fairly closely with the intermediate zone for the sands tested. Calculated ranges for coarse, medium and fine sands are superimposed on Fig. 14 to illustrate this point, using $R_{\mathrm{a}}=8 \mu \mathrm{m}$, a value quoted by Jardine et al. (1993) as typical for offshore piles. It is clear that values of $\delta_{\mathrm{p}}^{\prime} /\left(\phi_{\mathrm{ds}}^{\prime}\right)_{\mathrm{p}}$ for sand on steel can range between 0.5 and 1.0 , depending primarily on particle size. Silts may be expected to mobilise the full frictional resistance, unless the surface is particularly smooth. Gravels may be expected to show non-dilatant behaviour with low frictional resistance, unless the surface is particularly rough.

However, the clear pattern of behaviour described above may only be applicable at low stress levels. There is evidence from Fig. 8 that interface friction and dilation 

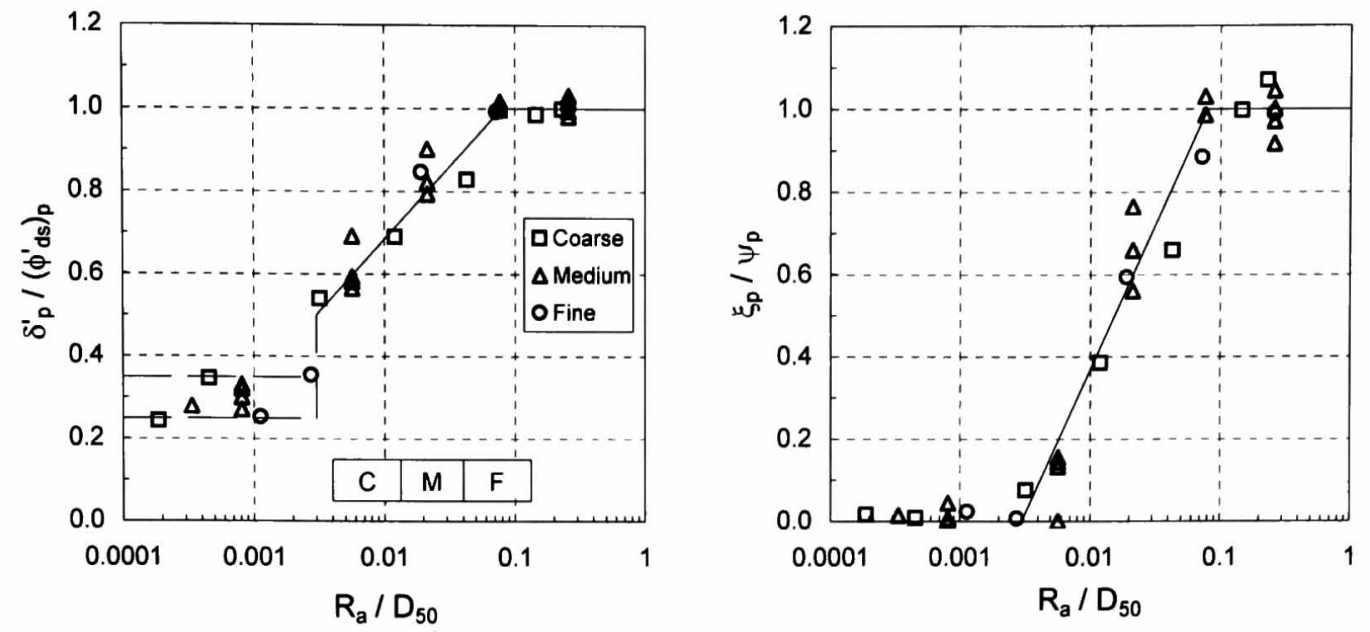

Fig. 14. Normalised interface friction and dilation vs. relative roughness (all tests at $25 \mathrm{kPa}$ )

values for tests on ALO and SIC cross over at higher stress levels, which will produce departures from the semi-logarithmic straight lines of Fig. 14.

\section{SUMMARY AND CONCLUSIONS}

A fundamental study of sand-steel interfaces has been carried out using a modified direct shear apparatus. The modifications allow the shear load to be applied at the sample centre, thereby enabling loads to be assessed correctly, and dilation to take place unimpeded. The symmetrical arrangement of Jewell and Wroth (1987) has been adopted, and other minor but significant modifications made to conventional practice. Three sands, one coarse, one medium and one fine, have been tested in conjunction with four steel surfaces and three sand surfaces of widely different roughnesses. Tests have explored a range of sand densities and normal stress levels.

Two different methods for quantifying roughness have been compared; normalised roughness $\left(R_{\max } / D_{50}\right)$ based on the maximum roughness, $R_{\max }$; and relative roughness $\left(R_{\mathrm{a}} / D_{50}\right)$ based on the average roughness, $R_{\mathrm{a}}$. The two methods are found to be equally good at unifying the data irrespective of particle size. The apparatus permits the accurate measurement of dilation, and both methods are also able to unify the dilation data.

When friction and dilation data are normalised by dividing by the values obtained at the same density in direct shear, the effects of density seemingly disappear, and unique relationships are found between the normalised friction and dilation data and relative roughness.

Stress-dilatancy analysis of peak friction and dilation angles from sand-on-steel interface tests suggests a relationship $\delta_{\mathrm{p}}^{\prime}=25^{\circ}+\xi_{\mathrm{p}}$ for all tests displaying dilation. This provides a clear rationale for dividing interface behaviour into two types: dilatant and non-dilatant, the former being characterised by particle rolling, the latter by particle sliding at the contact. For the test sands, the boundary between non-dilatant and dilatant behaviour occurs at a relative roughness, $R_{\mathrm{a}} / D_{50} \approx 0.003$. The interfaces become fully rough, mobilising the full strength of the sand, at $R_{\mathrm{a}} / D_{50} \approx 0.08$. Dilatant interfaces mobilise peak normalised interface friction ratios $\left(\delta_{\mathrm{p}}^{\prime} /\left(\phi_{\mathrm{ds}}^{\prime}\right)_{\mathrm{p}}\right)$ between 0.5 and 1.0 , which is the range of values likely to apply to sand on rolled steel surfaces.

The effect of stress level is different depending on the roughness of the surface. For dilatant interfaces, increasing stress levels result in reductions in peak interface friction and dilation. For non-dilatant interfaces, increasing stress levels result in increases in peak interface friction, thought to be due to increased ploughing.

\section{ACKNOWLEDGEMENTS}

The Authors record their thanks to Dr Thomas Pearce and Alan Speight of the Institute of Grinding Technology for the use of their profilometer; also to Mike Pope for technician support in the laboratory. Financial support from the Engineering and Physical Sciences Research Council, UK, is also gratefully acknowledged.

\section{NOTATION}

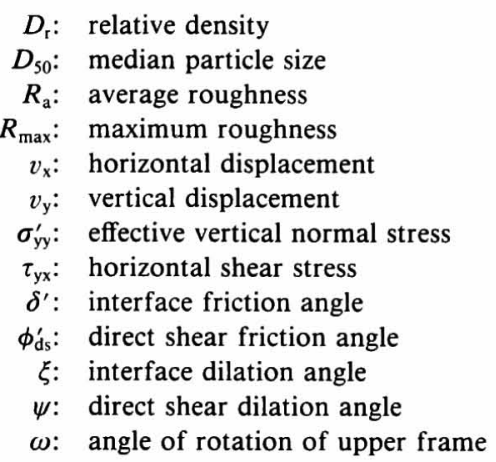

\section{REFERENCES}

1) Acar, Y. B., Durgunoglu, H. T. and Tumay, M. T. (1982): Interface properties of sand, J. Geotech. Engrg., ASCE, 108(4), 648-654.

2) Bolton, M. D. (1986): The strength and dilatancy of sands, Géotechnique, 36(1), 65-78

3) Butterfield, R. and Andrawes, K. Z. (1972): On the angles of 
friction between sand and plane surfaces, J. Terramechanics, 8(4), 15-23.

4) Dietz, M. S. (2000): Developing an holistic understanding of interface friction using sand within the direct shear apparatus, $P h D$ Thesis, University of Bristol.

5) Dove, J. E. and Jarrett, J. B. (2002): Behaviour of dilative sand interfaces in a geotribology framework, J. Geotech. Geoenviron. Engrg., ASCE, 128(1), 25-37.

6) Jardine, R. J., Lehane, B. M. and Everton, S. J. (1993): Friction coefficients for piles in sands and silts, Offshore Site Investigation and Foundation Behaviour, Society for Underwater Technology, 28, 661-677.

7) Jewell, R. A. and Wroth, C. P. (1987): Direct shear tests on reinforced sand, Géotechnique, 37(1), 53-68.

8) Kishida, H. and Uesugi, M. (1987): Tests of the interface between sand and steel in the simple shear apparatus, Géotechnique, 37(1), 45-52.

9) Lings, M. L. and Dietz, M. S. (2004): An improved direct shear apparatus for sand, Géotechnique, 54(4), 245-256.

10) Miura, K., Maeda, K. and Toki, S. (1997): Method of measurement for the angle of repose of sands, Soils and Foundations, 37(2), 89-96.

11) Miura, S. and Toki, S. (1982): A sample preparation method and its effects on static and cyclic deformation-strength properties of sand
Soils and Foundations, 22(1), 61-77.

12) Paikowsky, S. G., Player, C. M. and Connors, P. J. (1995): A dual interface apparatus for testing unrestricted friction of a soil along solid surfaces, Geotech. Test. J., GTJODJ, 18(2), 168-193.

13) Potyondy, J. G. (1961): Skin friction between various soils and construction materials, Géotechnique, 11(4), 339-353.

14) Stroud, M. A. (1971): The behaviour of sand at low stress levels in the simple shear apparatus, $P h D$ Thesis, University of Cambridge.

15) Subba Rao, K. S., Allam, M. M. and Robinson, R. G. (1996): A note on the choice of interfacial friction angle, Geotech. Engrg., Proc. Inst. Civ. Engrg., 119, 123-128.

16) Subba Rao, K. S., Allam, M. M. and Robinson, R. G. (1998): Interfacial friction between sands and solid surfaces, Geotech. Engrg., Proc. Inst. Civ. Engrg., 131, 75-82.

17) Uesugi, M. and Kishida, H. (1986a): Influential factors of friction between steel and dry sands, Soils and Foundations, 26(2), 33-46.

18) Uesugi, M. and Kishida, H. (1986b): Frictional resistance at yield between dry sand and mild steel, Soils and Foundations, 26(4), 139-149.

19) Uesugi, M., Kishida, H. and Tsubakihara, Y. (1988): Behaviour of sand particles in sand-steel friction, Soils and Foundations, 28(1), 107-118.

20) Yoshimi, Y. and Kishida, T. (1981): Friction between sand and a metal surface, Proc. 10th ICSMFE, 1, 831-834. 